07.2

\title{
Эффект саморазогрева в беспереходных вертикальных полевых транзисторах на основе структур „кремний на изоляторе“ с различной формой базы
}

\author{
(C) А.Э. Атамуратов ${ }^{1}$, Б.О. Жаббарова ${ }^{1}$, М.М. Халиллоев ${ }^{1}$, А. Юсупов ${ }^{2}$ \\ ${ }^{1}$ Ургенчский государственный университет, Ургенч, Узбекистан \\ ${ }^{2}$ Ташкентский университет информационных технологий им. Мухаммада аль-Хорезми, \\ Ташкент, Узбекистан \\ E-mail: atabek.atamuratov@urdu.uz, atabek.atamuratov@yahoo.com
}

Поступило в Редакцию 29 декабря 2020 г.

В окончательной редакции 8 марта 2021 г.

Принято к публикации 8 марта 2021 г.

\begin{abstract}
Моделируется эффект саморазогрева в наномасштабном беспереходном вертикальном полевом транзисторе, изготовленном на основе структур „кремний на изоляторе“ с поперечным сечением базы транзистора в форме прямоугольника, трапеции и треугольника. Показано, что для рассматриваемых структур температура в середине транзистора ниже, чем по его боковым граням около истока и стока. Помимо этого при прочих одинаковых условиях температура решетки зависит также от формы поперечного сечения базы.
\end{abstract}

Ключевые слова: эффект саморазогрева, температура решетки, теплопроводность, беспереходный FinFET-транзистор.

DOI: 10.21883/PJTF.2021.11.51003.18675

Одним из основных требований современной электроники является увеличение степени интеграции интегральных схем, что прямо связано с уменьшением или масштабированием размеров элементов. При этом транзисторы типа металл-оксид-полупроводник (МОП) являются наиболее широко используемыми. При масштабировании МОП-транзисторов возникают различные деградационные эффекты, среди которых можно отметить короткоканальные эффекты $[1,2]$, эффекты, приводящие к уменьшению надежности транзистора [3], эффект саморазогрева [4] и усиление чувствительности различных характеристик к встраиванию единичного заряда в подзатворном диэлектрическом слое или на границе подзатворного диэлектрика с каналом, что ведет к проявлению случайных телеграфных шумов в токе стока [5]. Кроме того, при изготовлении нанометровых плавниковых (вертикальных) транзисторов могут наблюдаться отклонения размеров, приводящие к изменению формы канала [6]. Для ослабления короткоканальных эффектов, возникающих в МОП-транзисторах при уменьшении их размеров до нанометровых масштабов, разработаны трехзатворные вертикальные полевые транзисторы (FinFET-транзисторы) [7]. Вслед за FinFET-транзисторами в качестве альтернативы для приборов с длинами канала менее $20 \mathrm{~nm}$ предложены беспереходные FinFET-транзисторы [1] на основе структур „кремний на изоляторе“ (КНИ), которые проявляют бо́льшую устойчивость к короткоканальным эффектам. Однако использование обратного оксидного слоя со сравнительно малым коэффициентом теплопроводности в технологии КНИ приводит к накоплению теплоты в канале и его разогреву при нанометровых размерах транзистора [8].
Увеличение температуры в канале (эффект саморазогрева) приводит к уменьшению тока стока и соответствующему ухудшению характеристик транзистора. Эффект саморазогрева и его зависимость от конфигурации и размеров различных слоев довольно хорошо изучался в FinFET-транзисторах, работающих в инверсной моде $[9,10]$, однако в беспереходных вертикальных МОПтранзисторах, работающих в аккумуляционном режиме, этот эффект изучался очень мало. Существуют работы, в которых предлагаются структуры беспереходных FinFET-транзисторов с особенностями для уменьшения эффекта саморазогрева $[11,12]$. В [11] рассматривается влияние профиля легирования канала на эффект саморазогрева, а в [12] показано уменьшение эффекта саморазогрева путем выбора определенного положения скрытого оксидного слоя и использования метода высокой концентрации носителей заряда вместо истоковых и стоковых областей в беспереходном FinFET-транзисторе.

В настоящей работе моделируется влияние эффекта саморазогрева на распределение температуры решетки вдоль канала беспереходного FinFET-транзистора на основе структур КНИ с различной формой базы транзистора. Рассматривались базы транзистора с формой поперечного сечения в виде треугольника, трапеции и прямоугольника (рис. 1, $a-c$ ).

3D-моделирование проводилось с использованием программы Advanced TCAD Sentaurus [13]. Наряду с моделью по умолчанию, в которой подвижность носителей принимается независимой и постоянной, использовались модели деградации подвижности, рассматривающие зависимость подвижности от концентрации легирования и учитывающие рассеяние на примесях и 

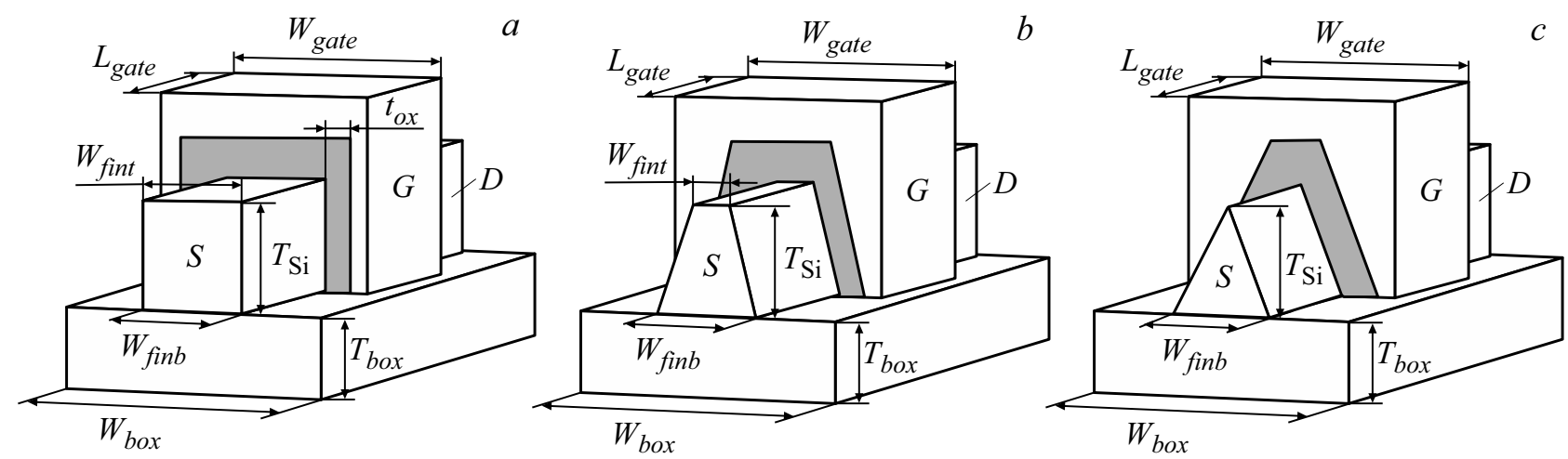

Рис. 1. Структуры моделируемого беспереходного КНИ FinFET-транзистора с различной формой базы: прямоугольное (a), трапецеидальное $(b)$ и треугольное $(c)$ поперечное сечение.

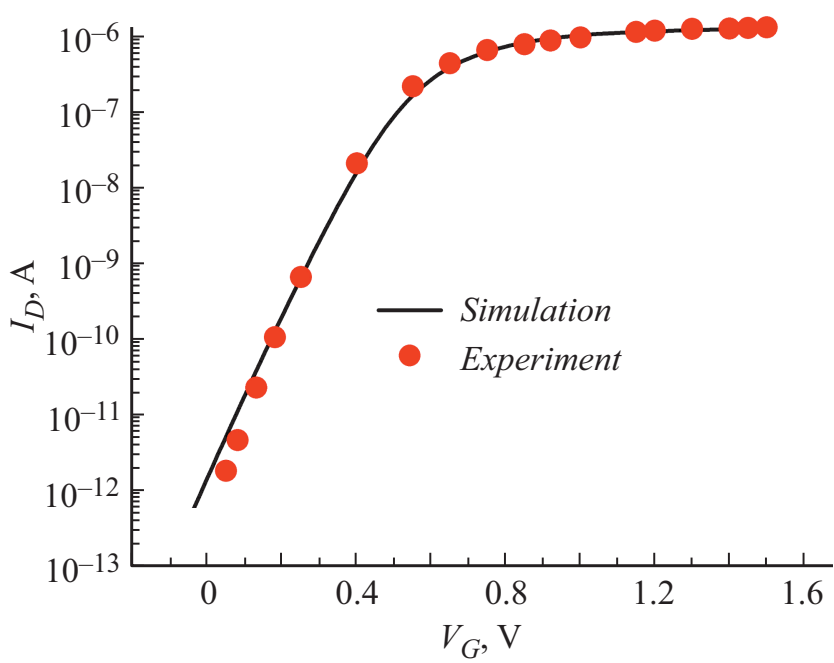

Рис. 2. Переходная вольт-амперная характеристика моделируемого транзистора и экспериментального образца $[15]$ с одинаковыми размерами и параметрами.

влияние высоких полей для учета эффекта насыщения скорости носителей, а также влияние нормальной составляющей поля для учета влияния границы на ток стока. Для учета эффекта саморазогрева были включены термодинамическая модель транспорта и модель рекомбинации Шокли-Рида-Холла. В связи с использованием диэлектрика с высокой диэлектрической проницаемостью в качестве подзатворного диэлектрика применялась соответствующая модель деградации подвижности. Поскольку размеры транзистора имеют нанометровые масштабы, необходимо принять во внимание и квантовые эффекты. Наиболее предпочтительной как при диффузионно-дрейфовом, так и при термодинамическом моделировании является квантовая корректировка по градиенту плотности [14], которая применялась в настоящей работе. Используемая модель калибровалась по экспериментальным результатам работы [15] (рис. 2).

При моделировании использовались следующие параметры структуры. Длина TiN-затвора $(G)$ выбиралась равной $10 \mathrm{~nm}$. В качестве подзатворного оксидного слоя взят $\mathrm{HfO}_{2}$, эквивалентная толщина которого составляла $t_{e f f}=0.9 \mathrm{~nm}$. Ширина скрытого оксидного слоя равна $W_{b o x}=69.4 \mathrm{~nm}$, а его толщина $-145 \mathrm{~nm}$. Параметром, отражающим форму базы транзистора, является ширина его потолка $W_{\text {fint }}$, которая для рассматриваемого трапецеидального сечения равна $10 \mathrm{~nm}$. База транзистора легирована бором с концентрацией $5 \cdot 10^{18} \mathrm{~cm}^{-3}$, имеет ширину у основания $W_{\text {finb }}=22 \mathrm{~nm}$ и толщину $T_{\mathrm{Si}}=9 \mathrm{~nm}$.

Результаты моделирования распределения температуры вдоль канала от истока до стока для нижней (кривая 1), средней (кривая 2) и верхней (кривая 3) частей базы транзистора по вертикали для транзистора с прямоугольным сечением представлены на рис. 3. Из результатов видно, что в середине вдоль канала температура ниже, чем по краям базы около истока и стока. Это связано со сравнительно малой теплопроводностью воздуха (от 0.0084 до $0.0915 \mathrm{~W} /(\mathrm{m} \cdot \mathrm{K}$ ) в диапазоне температур от 90 до 1473 K), окружающего кремниевую базу транзистора по боковым граням вблизи истока и стока, по сравнению с теплопроводностью подзатворного оксида гафния (от 0.49 до $0.95 \mathrm{~W} /(\mathrm{m} \cdot \mathrm{K})$ в диапазоне температур от 300 до $500 \mathrm{~K}$ ), покрывающего среднюю часть транзистора (канал), что приводит к разному теплоотводу вдоль всей кремниевой базы и соответствующему распределению температуры. Температуры в середине и на торцевых краях транзисторов различаются на $1-2 \mathrm{~K}$. Эти значения изменений температуры малы, но оценки по данным, приведенным в работе [10], показывают, что в надпороговой области изменения тока стока на каждый $1 \mu \mathrm{m}$ ширины канала при изменении температуры на $1 \mathrm{~K}$ составляют порядка $0.1 \mu \mathrm{A}$, что может быть важным при использовании транзисторов в схемах с малой потребляемой мощностью.

В боковых гранях базы транзистора контакт нижней части базы со скрытым оксидным слоем, теплопроводность которого находится в диапазоне от 7 до $13 \mathrm{~W} /(\mathrm{m} \cdot \mathrm{K})$, приводит к меньшей температуре в этой части, чем в окруженной воздухом верхней части 


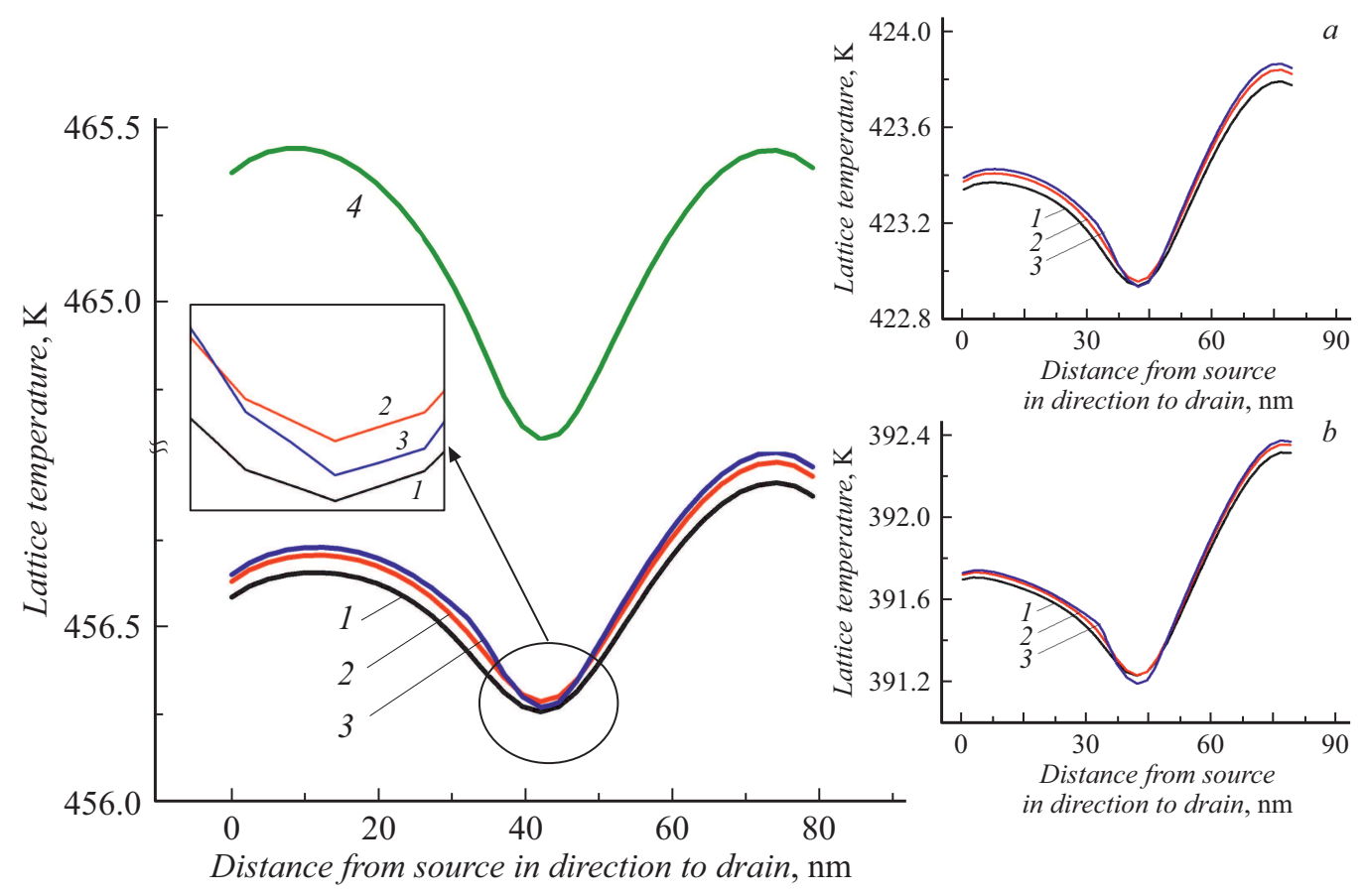

Рис. 3. Распределение температуры решетки вдоль канала от истока до стока в нижней (1), средней (2) и верхней (3) частях базы в беспереходном КНИ FinFET-транзисторе с прямоугольным поперечным сечением с $W_{\text {finb }}=22 \mathrm{~nm}$ и $T_{\mathrm{Si}}=9 \mathrm{~nm}$, а также для сравнения распределение температуры решетки вдоль середины канала в транзисторе с $W_{\text {finb }}=9 \mathrm{~nm}$ и $T_{\mathrm{Si}}=22 \mathrm{~nm}(4)$. На вставках показаны распределения температуры для транзисторов с трапецеидальным $(a)$ и треугольным $(b)$ сечениями канала. $V_{G}=1.6 \mathrm{~V}, V_{D}=0.75 \mathrm{~V}$.

со сравнительно малой теплопроводностью. Небольшой спад температуры по краям (около истока и стока) связан с отводом тепла через контакты.

В середине базы транзистора (в канале) более низкие температуры в нижней и верхней частях (рис. 3, кривые 1 и 3) по сравнению с температурой в середине по вертикали базы (рис. 3, кривая 2) связаны с расстоянием до теплоотводящих верхней и нижней граней базы.

Сравнение полученных результатов распределения температуры вдоль и поперек канала с результатами исследования эффекта саморазогрева в FinFET-транзисторе, работающем в инверсной моде [10], позволяет сделать вывод, что наблюдаемые различия в распределении температуры связаны не столько с наличием или отсутствием стоковых и истоковых областей, сколько с расположением и размерами контактов к этим областям. Эти контакты способствуют существенному теплоотводу и влияют на распределение температуры вдоль базы транзистора.

Для трапецеидального и треугольного поперечных сечений транзистора распределение температуры вдоль базы имеет тот же характер, что и для прямоугольного сечения (вставки $a$ и $b$ на рис. 3). Но при этом температура решетки в канале с прямоугольным сечением выше, чем для трапецеидального и треугольного сечений, а наименьшая температура наблюдается для треугольного сечения. Очевидно, что при больших сечениях при прочих одинаковых условиях через канал проходит больший ток и соответственно при этом выделяется большее количество теплоты, что приводит к нагреванию до более высоких температур. А для оценки влияния формы сечения было проведено сравнение распределения температуры в транзисторах с прямоугольными поперечными сечениями с $W_{\text {finb }}=22 \mathrm{~nm}, T_{\mathrm{Si}}=9 \mathrm{~nm}$ („горизонтальная“ структура) и с $W_{\text {finb }}=9 \mathrm{~nm}, T_{\mathrm{Si}}=22 \mathrm{~nm}$ („вертикальная“ структура), площади сечения которых равны (рис. 3). Из рисунка видно, что температуры в базе транзисторов различаются, хотя площади их сечения равны. Оценки на основе результатов моделирования распределения плотности тока по сечению канала показывают, что это связано со сравнительно большей средней плотностью тока в канале „вертикальной“ структуры, чем в случае „горизонтальной““.

Таким образом, результаты моделирования показывают, что в беспереходном КНИ FinFET-транзисторе температура в центре базы и распределение температуры вдоль канала зависят как от материала, окружающего базу, и его площади, так и от формы его поперечного сечения.

\section{Конфликт интересов}

Авторы заявляют, что у них нет конфликта интересов. 


\section{Список литературы}

[1] I. Ferain, C.A. Colinge, J.P. Colinge, Nature, 479 (7373), 310 (2011). https://doi.org/ 10.1038/ nature10676

[2] А.Э. Абдикаримов, А. Юсупов, А.Э. Атамуратов, Письма в ЖТФ, 44 (21), 22 (2018).

DOI: 10.21883/PJTF.2018.21.46852.17328

[Пер. версия: 10.1134/S1063785018110020].

[3] Y.-L. Yang, W. Zhang, S.-Y. Yan, Y.-H. Yu, Z.-Y. Fang, W.-K. Yeh, Vacuum, 181, 109601 (2020). https://doi.org/10.1016/j.vacuum.2020.109601

[4] W. Ahn, S.H. Shin, C. Jiang, H. Jiang, M.A. Wahab, M.A. Alam, Microelectron. Reliab., 81, 262 (2018). https://doi.org/10.1016/j.microrel.2017.12.034

[5] J.P. Campbell, L.C. Yul, K.P. Cheung, J. Qin, J.S. Suehle, A. Oates, K. Sheng, 2009 Proc. IEEE Int. Conf. on IC design and technology (ICICDT) (Austin, USA, 2009), p. 17. DOI: $10.1109 /$ icicdt.2009.5166255

[6] A. Abdikarimov, G. Indalecio, E. Comesana, N. Seoane, K. Kalna, A.J. Garsia-Lourero, A.E. Atamuratov, 17th Int. Workshop on computational electronics (IWCE 2014) (Paris, France, 2014), p. 247. DOI: 10.1109/iwce.2014.6865877

[7] J.P. Colinge, Microelectron. Eng., 84 (9-10), 2071 (2007). https://doi.org/ 10.1016/j.mee.2007.04.038

[8] D. Vasileska, S.M. Goodnick, K. Raleva, 13th Int. Workshop on computational electronics (Beijing, China, 2009). DOI: 10.1109/IWCE.2009.5091146

[9] G. Zhang, J. Lai, S. Zhu, S. Wei, F. Liang, C.-F. Yang, Microelectron. Reliab., 95, 52 (2019). https://doi.org/10.1016/j.microrel.2019.02.006

[10] R.P. Nelapati, K. Sivasankaran, Microelectron. J., 76, 63 (2018). https://doi.org/10.1016/j.mejo.2018.04.015

[11] H. Ferhati, F. Douak, F. Djeffal, Superlatt. Microstruct., 109, 869 (2017). DOI: 10.1016/ j.spmi.2017.06.009

[12] A. Kumar, A. Gupta, S. Rai, Int. J. Electron. Commun., 95, 162 (2018). https://doi.org/10.1016/j.aeue.2018.08.023

[13] http://www.synopsys.com

[14] A. Loureiro, N. Seoane, M.R. Aldegunde, R. Valin, A. Asenov, A. Martinez, K. Kalna, IEEE Trans. Comput.-Aided Des. Integr. Circuits Syst., 30 (6), 841 (2011). DOI: 10.1109/TCAD.2011.2107990

[15] S. Barraud, M. Berthomé, R. Coquand, M. Cassé, T. Ernst, M.-P. Samson, P. Perreau, K.K. Bourdelle, O. Faynot, T. Poiroux, IEEE Electron Dev. Lett., 33 (9), 1225 (2012). DOI: 10.1109/LED.2012.2203091 